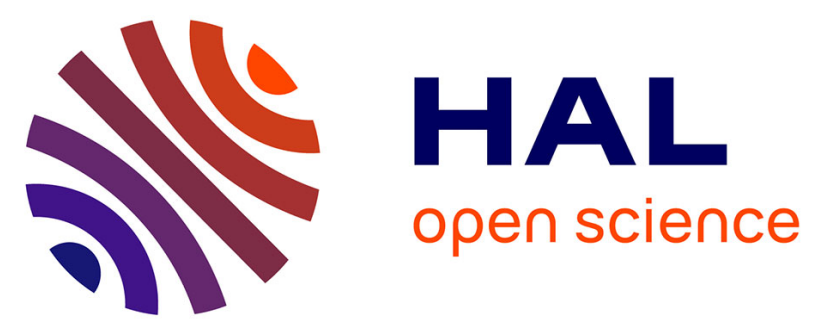

\title{
3D-printed, carbon-based, lossy photonic crystals: Is high electrical conductivity the must?
}

Polina Kuzhir, Alesia Paddubskaya, Dzmitry Bychanok, Aleksandr Liubimau, Alberto Ortona, Vanessa Fierro, Alain Celzard

\section{- To cite this version:}

Polina Kuzhir, Alesia Paddubskaya, Dzmitry Bychanok, Aleksandr Liubimau, Alberto Ortona, et al.. 3D-printed, carbon-based, lossy photonic crystals: Is high electrical conductivity the must? Carbon, 2021, 171, pp.484-492. 10.1016/j.carbon.2020.09.020 . hal-03271477

\section{HAL Id: hal-03271477 \\ https://hal.univ-lorraine.fr/hal-03271477}

Submitted on 28 Jun 2021

HAL is a multi-disciplinary open access archive for the deposit and dissemination of scientific research documents, whether they are published or not. The documents may come from teaching and research institutions in France or abroad, or from public or private research centers.
L'archive ouverte pluridisciplinaire HAL, est destinée au dépôt et à la diffusion de documents scientifiques de niveau recherche, publiés ou non, émanant des établissements d'enseignement et de recherche français ou étrangers, des laboratoires publics ou privés.

\section{(1) (1) $\$$}

Distributed under a Creative Commons Attribution - NonCommercial - NoDerivatives| 4.0 


\title{
3D-printed, carbon-based, lossy photonic crystals: is high electrical conductivity the must?
}

\author{
Polina Kuzhir ${ }^{1,2}$, Alesia Paddubskaya ${ }^{2}$, \\ Dzmitry Bychanok ${ }^{2,3}$, Aleksandr Liubimau ${ }^{4,2}$,
}

Alberto Ortona $^{5}$, Vanessa Fierro ${ }^{6}$ and Alain Celzard ${ }^{6 *}$

${ }^{1}$ Institute of Photonics, Department of Physics and mathematics, University of Eastern Finland, Yliopistokatu 7, FI-80100 Joensuu, Finland

${ }^{2}$ Institute for Nuclear Problems, Belarusian State University, 220006 Minsk, Belarus

${ }^{3}$ Tomsk State University, 36, Lenin Avenue, Tomsk, 634050, Russia

${ }^{4}$ Belarusian State Technological University, Sverdlova 13a, Minsk 220006, Belarus

${ }^{5}$ MEMTi, SUPSI, Via Cantonale 2C, CH-6928 Manno, Switzerland

${ }^{6}$ Université de Lorraine, CNRS, IJL, F-88000 Epinal, France

* Corresponding author. E-mail address: alain.celzard@univ-lorraine.fr (A. Celzard) 


\begin{abstract}
The electromagnetic response of 3D-printed periodic carbon structures was investigated numerically and experimentally in the microwave $(26-37 \mathrm{GHz})$ and terahertz $(0.2-1.2 \mathrm{THz})$ frequency ranges. The reflection, transmission and absorption spectra, as well as the effects of the concentration of electromagnetic waves, were analysed and discussed. High broadband absorption was observed for the 3D-printed cellular structures based on a moderately conductive $\left(1-30 \mathrm{~S} \cdot \mathrm{m}^{-1}\right)$ skeleton, whereas perfect tuneable resonant absorption could be achieved by 3D meshes made of highly conductive $\left(1200-2000 \mathrm{~S} \cdot \mathrm{m}^{-1}\right)$ glassy carbon. We show that laser stereolithography (SLA) or fused deposition modelling (FDM) 3D-printing technique should be preferred for getting pre-defined required electromagnetic performances depending on the intended application.
\end{abstract}




\section{Introduction}

The problems of periodic concentration of the electromagnetic (EM) field as well as the perfect absorption of microwaves and $\mathrm{THz}$ radiation are among the hottest issues of electromagnetics nowadays. These two questions are indeed expected to lead to a scientific breakthrough and to be related to security devices, such as ultra-sensitive detectors of EM pulses and continuous radiation, EM wave concentrators, compact waveguides, and even unconventional EM memory and logic. There are many different routes for producing perfect absorbers, whether based on bulk materials exploring dielectric [1] and magnetic [2] losses, on porous structures [3-5], on thin film effect [6,7], or on Salisbury screen [8,9]. Metamaterials and lossy photonic crystals are the most advantageous systems if a spatial periodic distribution of EM field is needed [10-12].

To date, many technologies are known for the practical production of photonic crystals. These are, to name a few, electrochemical fabrication of porous silicon, alumina or III-V semiconductors; micro and nanofabrication of metallic nanoparticle or nanowire arrays for waveguiding plasmon polaritons; electron beam, interference and holographic lithographies; molecular beam epitaxy (see [13] for a review). To achieve adjustability, spatially periodic liquid crystals and colloidal crystals have been proposed $[13,14]$. At the same time, the possibilities offered by regular meshes produced by a simple technological route, additive manufacturing, were mentioned in a very few publications only [15-17]. Lossy tuneable photonic crystals that would be 3D-printed, and therefore easy to reproduce and implement, might solve a number of materials-related problems in modern applied electromagnetics.

There are at least two routes to produce electrically conductive 3D periodic structures using additive manufacturing. The first one (approach (i) in Fig.1), described in detail in a recent paper from our group [17], consists in producing a polymeric 3D mesh by laser stereolithography (SLA) based on photocurable polymers. As the latter have a negligible carbon yield upon pyrolysis, the structure is firstly impregnated with a phenolic resin under hydrothermal 
conditions in the presence of a nickel salt acting as graphitisation catalyst. After carbonisation and removal of nickel by acid leaching, the 3D structure is converted into a glassy carbon periodic mesh having an excellent electrical conductivity. Playing with the precursor's chemical nature, template geometry and pyrolysis conditions truly allows producing any 3D all-carbon architecture whose backbone presents conductivities in the typical range $2000-20000 \mathrm{~S} \cdot \mathrm{m}^{-1}$.

\section{Approach (i): All-carbon 3D periodic lattice}

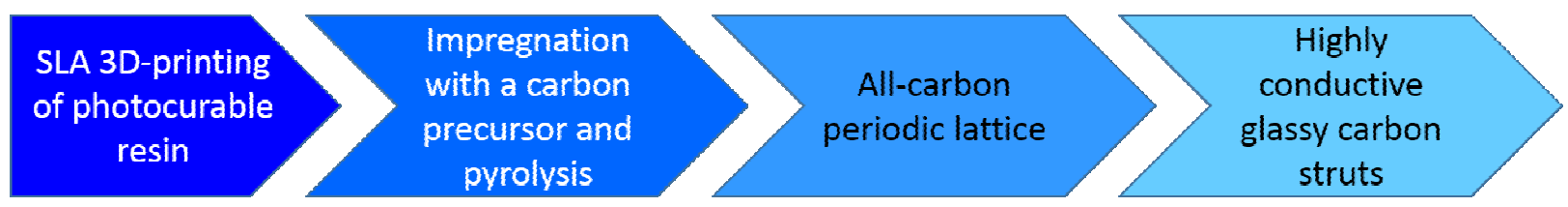

\section{Approach (ii): Carbon-filled composite 3D periodic lattice}

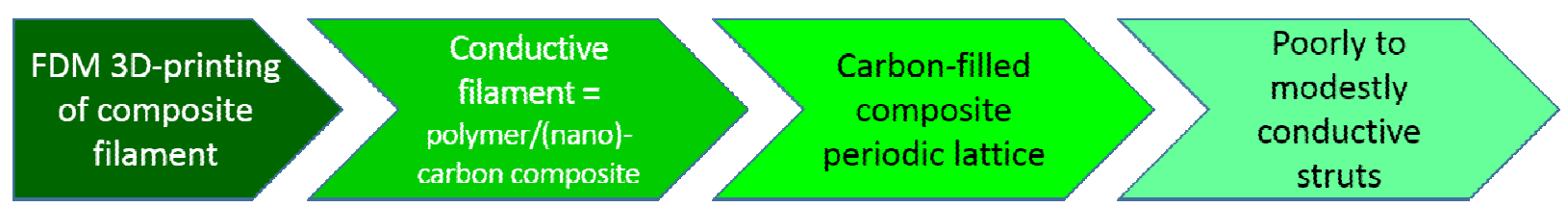

Figure 1. Schematic representation of two basic approaches for 3D-printing electrically conductive, carbon-based periodic lattices.

Another possibility (approach (ii) in Fig. 1) is to print 3D architectures by the fused deposition modelling (FDM) technique, using a conductive composite such as PLA or ABS filled with graphene nanoplatelets for instance $[18,19]$. Obviously, unlike the SLA technique, no pyrolysis is possible afterwards, and therefore the electrical conductivity of the skeleton is limited by that of the composite filament used to build the structure, within the typical range 0.1 - $200 \mathrm{~S} \cdot \mathrm{m}^{-1}$. Additionally, only simple porous architectures are possible, since the molten filament needs to be deposited on a support material, hence generally on itself. This drawback can be overcome, however, with special 3D-printers that allow the use of a second polymer acting as support, which can be leached out after 3D printing for instance. 
The present paper deals with the electromagnetic properties of 3D-printed structures, having the same geometry but prepared by SLA or FDM techniques. The different corresponding characteristics are highlighted, and the pros and cons from the electromagnetic point of view are discussed.

\section{Materials and methods}

\subsection{Modelling the electromagnetic properties of carbon-based 3D periodic structures}

Herein, two types of cellular architectures have been considered. Both have a geometry made of open cells, of either Kelvin (tetrakaidecahedron) or Gibson-Ashby (cubic) type, connected with each other through windows as shown in Fig. 2. The size of the windows connecting the cells, here called $a$, depends on cell size and strut thickness. Gibson-Ashby cells thus have one single window size, and the corresponding lattice parameter is $L$, see Fig. 2(a). Concerning Kelvin cells, two different windows exist, which might be important to take into account for the electromagnetic analysis, and $a$ in Fig. 2(b) refers to the largest one; $L$ is again the lattice parameter. The ideal structures in Fig. 2 are based on struts of equal length $l$, but in practice, and because of the shrinkage occurring during pyrolysis, $l$ may vary from one material to another (see below).

The CST Studio® 3D electromagnetic simulation software was used for modelling the electromagnetic response of these carbon-based cellular structures, using the finite integration technique (FIT) applicable for both time domain and frequency domain. FIT is a discretisation scheme for integral Maxwell's equations, where the resulting matrix equations of the discretised fields are used for numerical EM simulations (see [20] for details of calculations and simulations). 


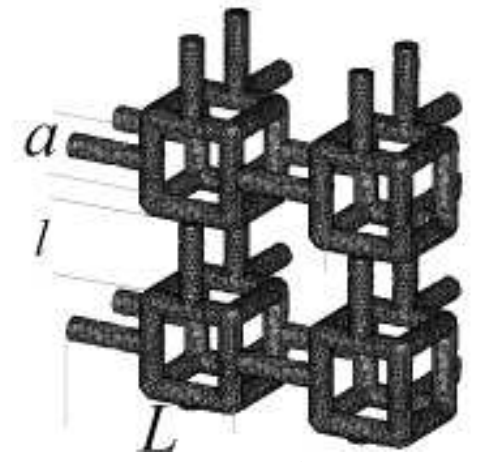

a)

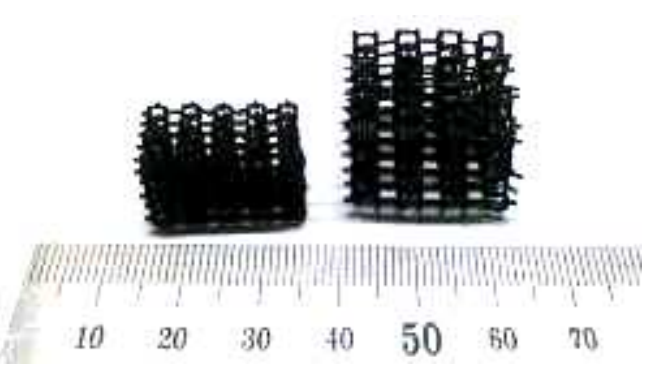

c)

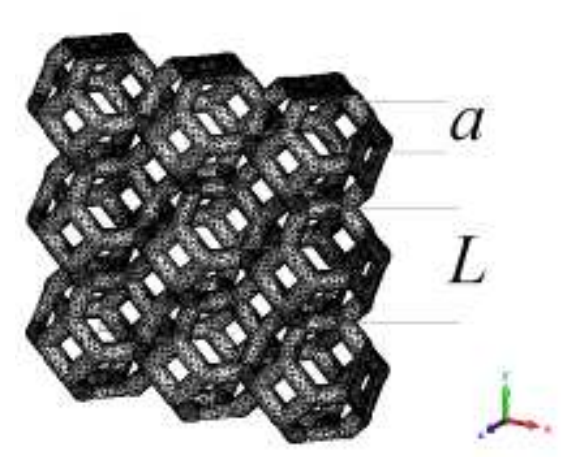

b)

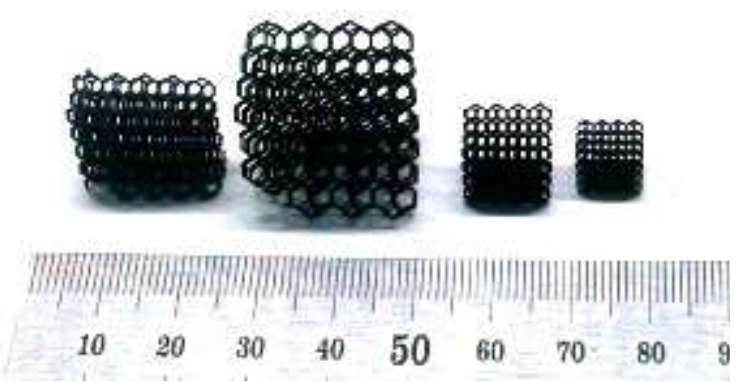

d)

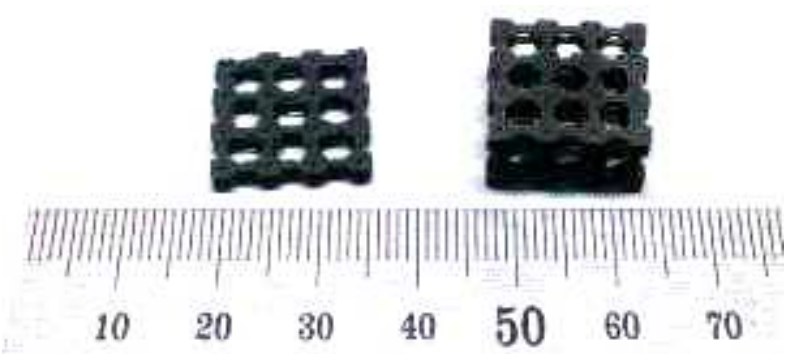

e)

Figure 2. Schematic presentation of unit cells of the 3D periodic structures investigated here: (a) Gibson-Ashby and (b) Kelvin cells. Samples produced by SLA and made of pure glassy carbon of high conductivity, and corresponding to (c) Gibson-Ashby cells (left: sample S; right: sample L, see Table 1 for details), and (d) Kelvin cells (from left to right: samples A5Ni, B5Ni, C5Ni and D5Ni, see Table 1 for details). (e) Samples produced by FDM and made of composite skeleton of moderate conductivity. The rulers shown in photos (c), (d) and (e) are graduated in centimetres.

The simulation of microwave and $\mathrm{THz}$ responses was carried out for both Kelvin- and Gibson-Ashy-type 3D structures with geometric parameters (i.e., cell and window sizes) compatible with the considered wavelengths. The simulation details (type of unit cell, lattice parameter, strut thickness, and number of cell layers in the investigated structure) are given in 
the corresponding figure captions below. The electromagnetic response in the frequency range from $\mathrm{cm}$ to sub-mm were studied as a function of cell size, strut thickness and backbone electrical conductivity, number of cell layers in the 3D structure and relative permittivity of the surrounding dielectric medium.

\subsection{Preparation of $3 D$ periodic architectures, materials structure and properties}

\subsubsection{Highly-conductivity skeleton}

The details of the preparation of 3D periodic glassy carbon (see Fig. 2(c) and 2(d)) by the SLA technique can be found elsewhere [17]. In short, polymer templates were 3D-printed with an Objet Eden $260 \mathrm{~V}$ 3D prototyping machine using Fullcure 705 and 850 VeroGray (Objet Ltd.) photosensitive resins composed of acrylics, urethanes and epoxies. The latter contained a photoinitiator that triggered the polymerisation under UV light. After curing, the polymer meshes were washed with ethanol, dried, and then soaked in an aqueous solution containing $0.475 \mathrm{~g}$ of resorcinol, $0.7 \mathrm{~g}$ of a $37 \mathrm{wt} \%$ formaldehyde solution, and $22 \mathrm{~g}$ of nickel nitrate hexahydrate. The whole was subjected to hydrothermal conditions $\left(150^{\circ} \mathrm{C}, 24 \mathrm{~h}\right.$, self-generated pressure $)$ in an autoclave. After cooling, the 3D structures were washed with water, dried and pyrolysed at $1000^{\circ} \mathrm{C}\left(1^{\circ} \mathrm{C} \min ^{-1}, 2 \mathrm{~h}\right)$, then washed with 37 wt. $\% \mathrm{HCl}$ and then with water, and dried. The resultant periodic cellular glassy carbon replicas presented a carbon yield and a volume shrinkage of $20 \%$ and $72 \%$, respectively. The main geometric features as well as the name of the samples (from our previous work [17]) are presented in Table 1. The electrical conductivity of the struts of the 3D structures printed by FDM and SLA techniques were $10 \mathrm{~S} \mathrm{~m}^{-1}$ and $1200-$ $2000 \mathrm{~S} \mathrm{~m}^{-1}$, respectively.

Table 1. Geometric parameters of the glassy carbon 3D periodic structures based on Kelvin and Gibson-Ashby cells: strut length $l$, strut thickness $d$, and lattice parameter (cell size) $L$.

\begin{tabular}{cccc}
\hline Sample name & $l(\mathrm{~mm})$ & $d(\mathrm{~mm})$ & $L(\mathrm{~mm})$ \\
\hline B5Ni & 1.9 & 0.5 & 5.37
\end{tabular}




\begin{tabular}{cccc} 
A5Ni & 1.3 & 0.5 & 3.67 \\
C5Ni2 & 1.0 & 0.5 & 2.82 \\
D5Ni4 & 0.7 & 0.5 & 1.97 \\
Gibson-Ashby-L & 2.0 & 0.7 & 7.0 \\
Gibson-Ashby-S & 1.0 & 0.7 & 3.6 \\
\hline
\end{tabular}

\subsubsection{Low-to-medium conductivity skeleton}

CYCOLAC ${ }^{\mathrm{TM}}$ RESIN MG47F (ABS) polymer was used as a matrix for the manufacture of 3D printable filament. Milled carbon fibres supplied by Svetlogorskkhimvolokno (Belarus) have been used as conductive additives. The supplier provided the following characteristics for the fibres: carbon content 95 to $97 \%$, packing density $0.205 \mathrm{~g} \mathrm{~cm}^{-3}$, specific fiber density $1.45-1.50$ $\mathrm{g} \mathrm{cm}^{-3}$, fiber diameter 5 to $7 \mu \mathrm{m}$.

The preparation of ABS-based filaments with carbon filler contents of 6 and 12 wt. \% was carried out by melt mixing using a Rondol twin-screw extruder (screw diameter of $10 \mathrm{~mm}$ ) in two stages: (i) production of a homogeneous compound; and (ii) drawing of a filament from the compound. Before extrusion, the $\mathrm{ABS}$ and the carbon material were dried at $80^{\circ} \mathrm{C}$ for 4 hours. The processing was done at $180-210^{\circ} \mathrm{C}$, depending on the zone of the extruder, at a screw speed of $70 \mathrm{rpm}$ and a head pressure of 50-60 bar. The nominal diameter of the die was $1.78 \mathrm{~mm}$. The filament diameter was $1.75 \pm 0.05 \mathrm{~mm}$.

Then, samples in the form of Gibson-Ashby cells were printed using the Creatbot F430 printer with the following experimental parameters: nozzle diameter $0.4 \mathrm{~mm}$, layer thickness 0.2 $\mathrm{mm}$, printing speed $10 \mathrm{~mm} \mathrm{~s}^{-1}$, filling density $100 \%$, printing temperature $235^{\circ} \mathrm{C}$, table temperature $95^{\circ} \mathrm{C}$ and chamber temperature $60^{\circ} \mathrm{C}$.

The following geometric parameters were used: $a=0.85 \mathrm{~mm}$ and $L=2.4 \mathrm{~mm}$, to compare the modelling and the experimental results. The thickness of the skeleton have being chosen as an example of one of the most often used in simple industrially available regular architectures 
made by FDM technology. The conductivity of the composite skeleton made of 6 and 12 wt. \% of carbon filler in ABS was 0.5 and $10 \mathrm{~S} \cdot \mathrm{m}^{-1}$, respectively.

\subsection{Measurement of electrical and electromagnetic properties of $3 D$ periodic structures}

The $d c$ electrical conductivity of the sample's backbone was measured using the four-point method with a Keithley 2636 B source-meter. For that purpose, 10 homogeneous cylindrical parts of the backbone, i.e., 10 struts were detached from the 3D structures and their ends were covered with silver paint. Such contacts were used for carrying current, whereas the voltage drop was measured between $20 \mu \mathrm{m}$-thin tungsten needles accurately positioned by use of a MicroXact manual probe station. The average dc conductivity was then calculated.

For microwave measurements in the frequency range $25.96-37.5 \mathrm{GHz}$, the samples were placed directly in a waveguide of cross-section $7.2 \times 3.4 \mathrm{~mm}$, perpendicular to the wave propagation. Then, an ELMIKA R2-408R panoramic analyser of VSWR (standing wave ratio by voltage) was used to measure the modules of the amplitude of transmitted $\left(S_{21}\right)$ and reflected $\left(S_{11}\right)$ signals. Above $100 \mathrm{GHz}$, an EKSPLA T-Spec terahertz time-domain spectrometer was used to carry out time-resolved $\mathrm{THz}$ spectroscopy in the frequency range from 0.1 to $2 \mathrm{THz}$. The spectral resolution was better than $5 \mathrm{GHz}$, and the signal-to-noise ratio was higher than 106:1 at 0.4 THz. The accuracy of the measurements in the transmission mode is $10^{-5}$, whereas in reflection mode it is $10^{-4}$.

\section{Results and discussion}

\subsection{D regular meshes: large vs low-to-medium conductivity}

The first fundamental question that has been addressed is the influence of the skeleton electrical conductivity on the EM performances of carbon-based 3D periodic structures. Let us consider the case of a highly conductive skeleton, i.e., $2000 \mathrm{~S} \cdot \mathrm{m}^{-1}$. According to the finite element modelling results, see Fig. 3(a), the cellular structure composed of two layers of cubic 
cells is fully reflective in the entire range of investigated frequencies, except in resonant conditions for which the wavelength is close to the cell size. In such conditions, the carbon porous structure behaves as a photonic crystal and becomes perfectly absorptive.

Moreover, the position of the absorption peak can be easily shifted by changing the relative permittivity of the dielectric medium surrounding the carbon struts, for instance from $\varepsilon=1$ to 3 . This can be easily achieved experimentally by impregnating the porous structures with a dielectric liquid or a polymer instead of letting the carbon struts in air. Under these conditions, the frequency of the absorption peak decreases by a factor 2 to 3 , i.e., from $70-100 \mathrm{GHz}$ to 35 GHz. In practice, the permittivity of non-polar gases/liquids depends on the temperature, and this effect can be used for tuning even more precisely the frequency of the absorption peak. Indeed, increasing the temperature of the dielectric medium results in a decrease in the number of molecules per unit volume, and hence the decrease in density produces the decrease in the relative permittivity of the impregnation medium.

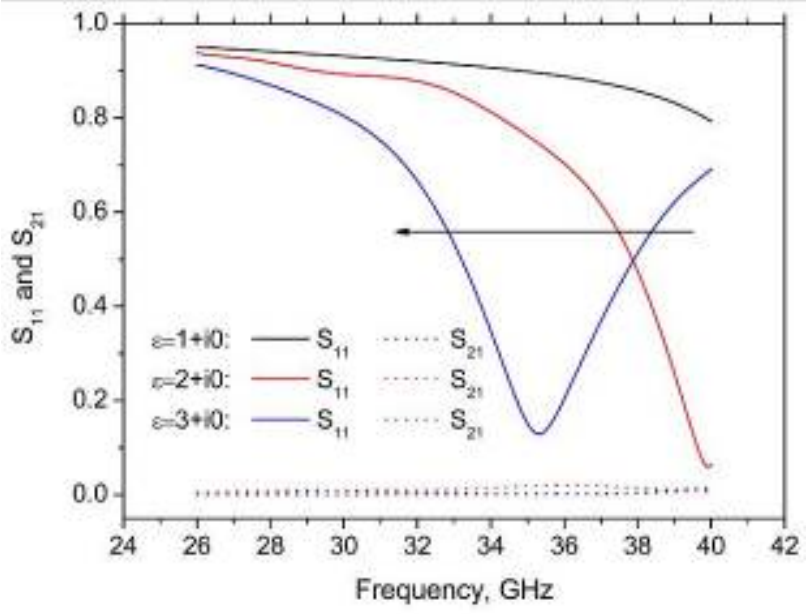

a)

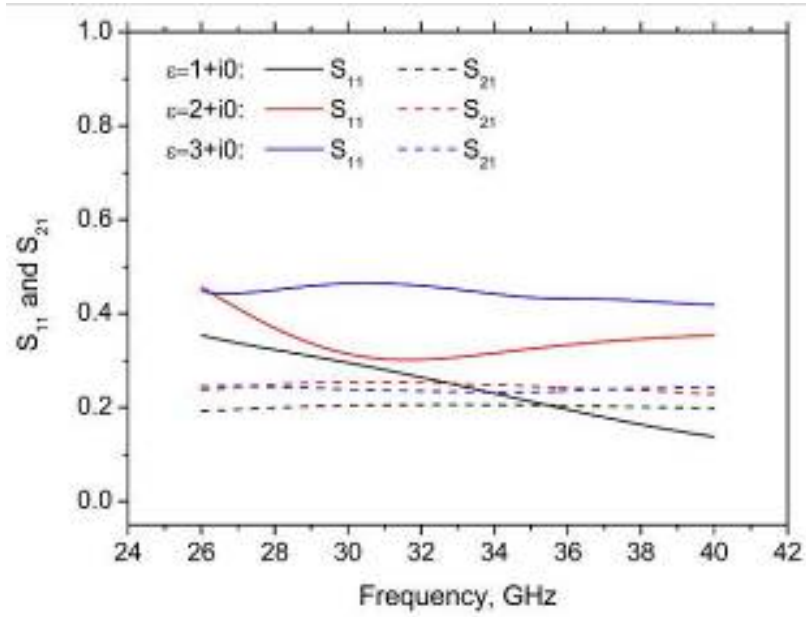

b)

Figure 3. Frequency dependence of the $S$-parameters for one layer of Gibson-Ashby cells such that $L=2.4 \mathrm{~mm}$ and $a=0.84 \mathrm{~mm}$, for different relative permittivity values $\varepsilon$ of the impregnation medium and two values of dc electrical conductivity of the carbon backbone: (a) $2000 \mathrm{~S} \mathrm{~m}^{-1}$; and (b) $10 \mathrm{~S} \mathrm{~m}^{-1}$.

Interestingly, the 3D architecture having the same geometry but a low-to-medium electrical conductivity, $10 \mathrm{~S} \cdot \mathrm{m}^{-1}$, did not demonstrate any perfect resonant absorption, see again Fig. $3 \mathrm{~b}$. 
Impregnating such poorly conductive structure with various dielectric media is therefore not expected to tune the EM response. Moreover, using dielectrics with a permittivity higher than that of air should suppress the absorption ability of these 3D-printed materials.

However, the case of rather low backbone dc conductivity is favourable when considering their high broadband absorption. Unlike 3D meshes based on glassy carbon, poorly conductive structures provide high (up to 85-94\%) but not total absorption in the overall microwave frequency range, see Fig. 4. In contrast, a noteworthy feature of the EM response of non-highly conductive 3D architectures is the existence of an optimal conductivity of the backbone, corresponding to the particular lattice parameters at which an almost perfect broadband absorption can be achieved. For instance, in the case of cubic cells with the parameters presented in the caption of Fig. 4, no truly high conductivity is required. The optimal conductivity is indeed as low as $10 \mathrm{~S} \cdot \mathrm{m}^{-1}$, and increasing further the backbone conductivity at fixed geometry leads to a lower absorbance, e.g. $60 \%$ at $100 \mathrm{~S} \cdot \mathrm{m}^{-1}$.

The experimental data proving the modelling results for the 3D-printed FDM cubic cell structure composed of two layers made of filament having a conductivity of 0.5 and $10 \mathrm{~S} \cdot \mathrm{m}^{-1}$ are presented in Fig. 4c. One can see that, in agreement with the simulation results for the $10 \mathrm{~S} \cdot \mathrm{m}^{-1}$ filament, the absorption reaches $80 \%$ at $30 \mathrm{GHz}$. 


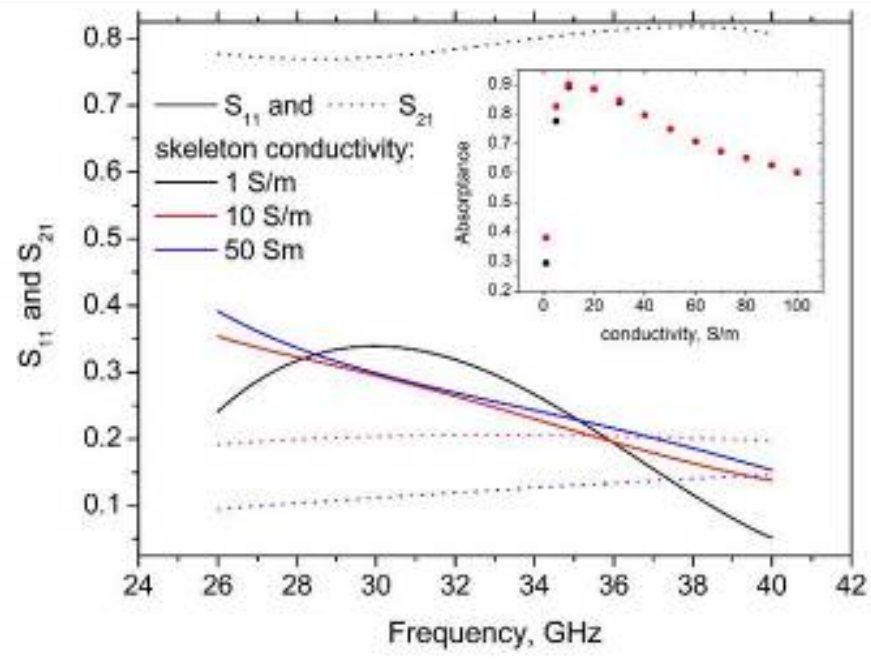

a)

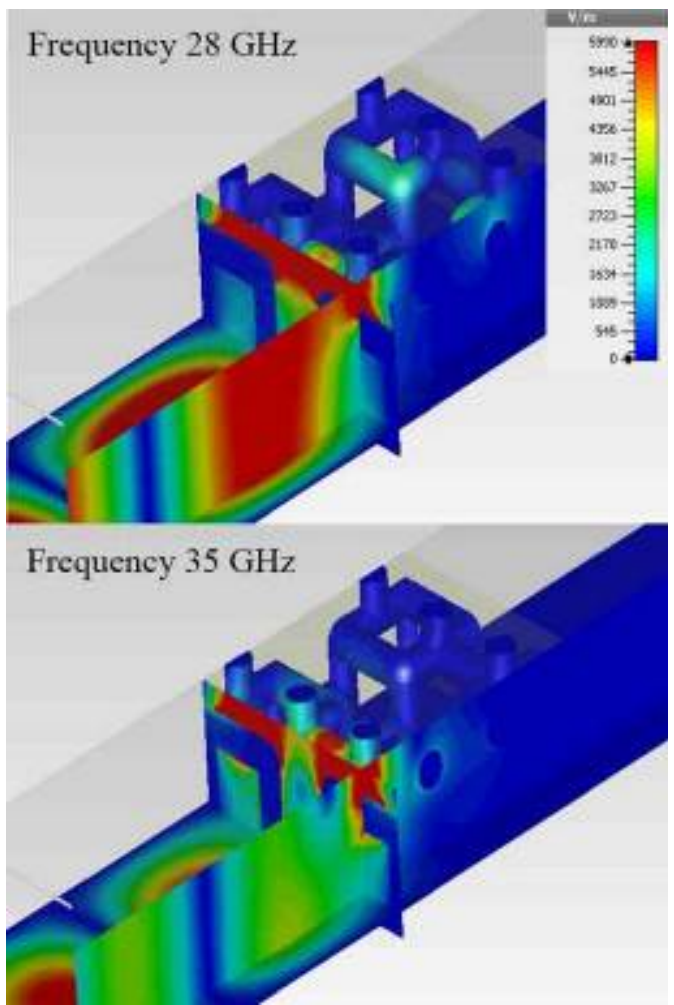

b)

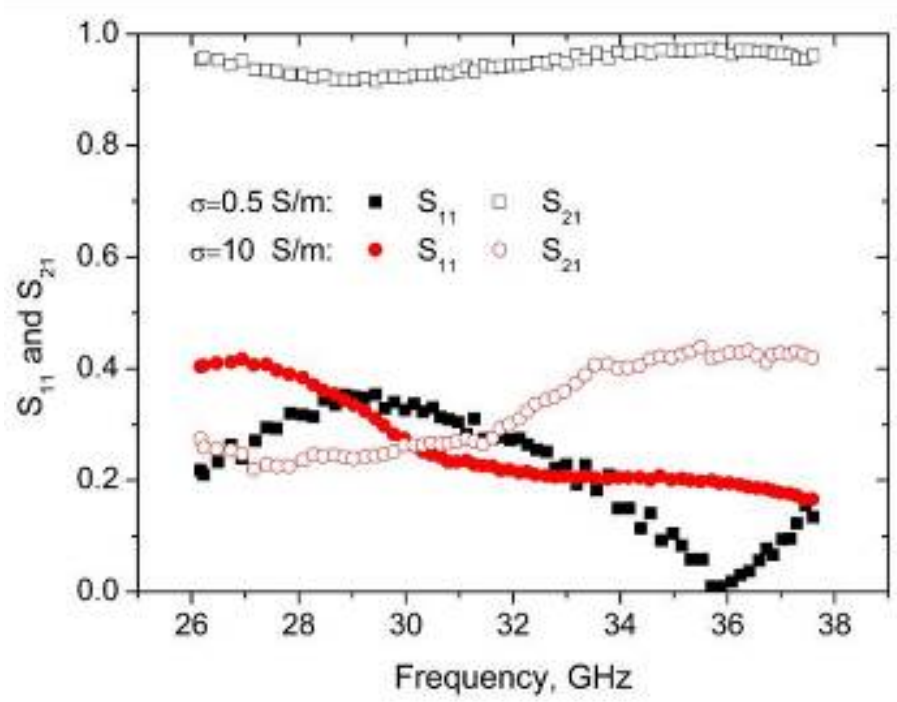

c)

Figure 4. (a) $S$-parameters of a single-layer of Gibson-Ashby cells having the three different conductivities indicated on the plot, as a function of frequency. In the numerical calculation (see field distribution in (b)), the following parameters were used: $a=0.85 \mathrm{~mm}, L=2.4 \mathrm{~mm}$, and dielectric constant of the medium surrounding the structure $\varepsilon=1$. The inset in (a) shows the dependence of the absorbance as a function of the backbone electrical conductivity; (c) Results of experimental measurements of a two-layer cubic cell structure in the waveguide. 
What is also important is that depending on the geometry of the regular architecture, including the skeleton thickness, size and number of the open cells and windows, numbers of layers in the mesh one may approach to the high absorption ability playing with the conductivity of the material the skeleton made of. Or vice versa (this situation is more realistic for the industrial applications) having the filament of particular conductivity (e.g. in the range from 5 to $50 \mathrm{~S} / \mathrm{m}$ ) one always may propose through the modeling steps the particular simple geometry for 3D printer, which will allow getting highly lossy photonic crystal.

Figure 4a inset presents the calculated by FEM modelling and experimentally measured absorption ability of a single-layer of Gibson-Ashby cells as a function of the backbone electrical conductivity. It can be easily seen that the same structure (having the same geometry) could provide absorption as high as $80 \%$ when the conductivity of the backbone is varied from 5 to 40 $\mathrm{S} / \mathrm{m}$.

The second question to address is the influence of the window size on the EM response of the 3D structures. For investigating this point, the electrical conductivity of the backbone was set constant and all the geometric parameters were fixed except the strut thickness. Fig. 5 thus shows how the EM response varies when the thickness of the struts is changed from 0.5 to $1 \mathrm{~mm}$. As in the case of conductivity in Fig. 4, there is an optimal window size, all other things being constant, which leads to a high absorption ability of the structure in a wide frequency range. In the present case, it corresponds to the intermediate backbone thickness, $0.84 \mathrm{~mm}$. 


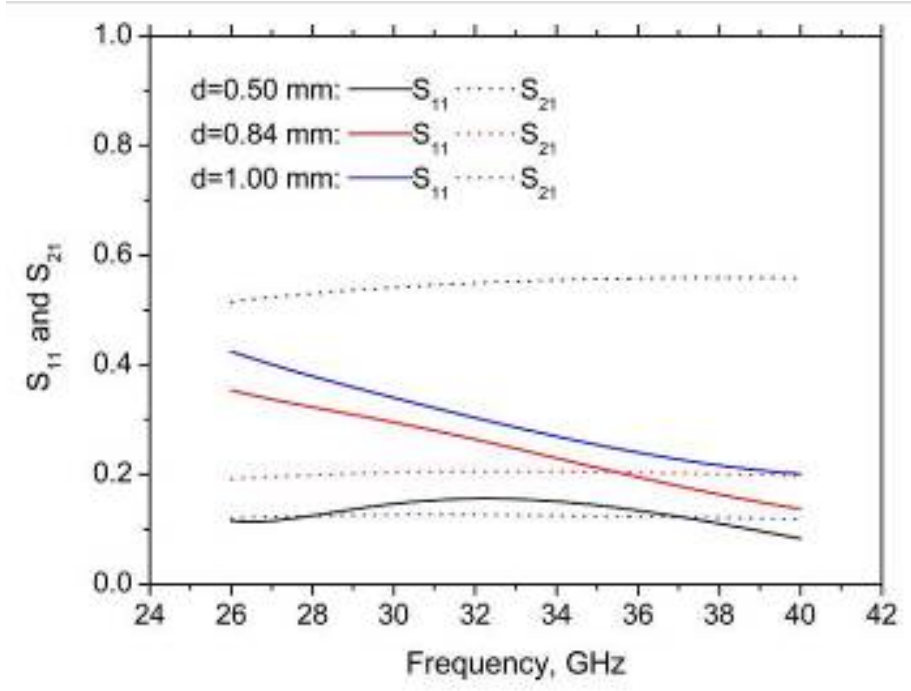

Figure 5. $S$-parameters of two layers of Gibson-Ashby cells having the three different strut thicknesses $(d)$ indicated on the plot, as a function of frequency. In the numerical calculation, the following parameters were used: backbone dc conductivity $10 \mathrm{~S} \mathrm{~m}^{-1}, L=2.4 \mathrm{~mm}$, and $\varepsilon=1$.

Fig. 6 illustrates the periodic concentration of the electromagnetic field within the Kelvintype cells at the resonance frequency, whereas this 3D structure is fully reflective for all other frequencies. This effect is due to the matching of the corresponding resonant wavelength with the cell parameters. Thus, changing the window size and keeping the same cell size also produces significant changes in the electromagnetic response. It suggests that one may tune smoothly the EM behaviour of the 3D periodic structure by mechanical deformation. This can be experimentally achieved if, for instance, the structure is built with a soft polymer filled with conductive nanoparticles above the percolation threshold [21].

In addition to mechanical robustness, Kelvin cells have one more advantage with respect to Gibson-Ashby cells. Indeed, there are only two free parameters for one given backbone conductivity (cell size and strut thickness) in cubic cells, whereas there is one additional degree of freedom (two different window sizes instead of just one) in Kelvin cells. As any geometric feature must have the corresponding resonance in case of high conductivity, one may also expect the resonance of Kelvin cells in the infrared range, as already observed for reticulated vitreous carbon foams [5]. 


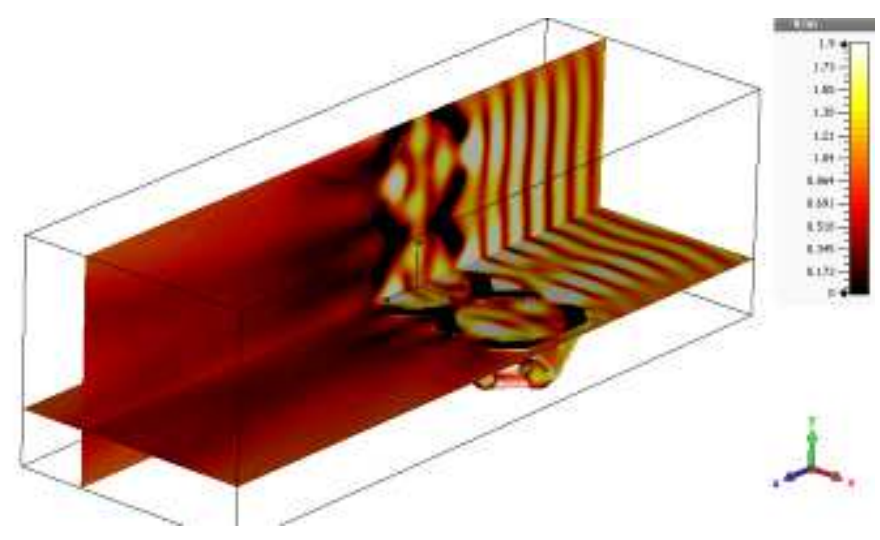

a)

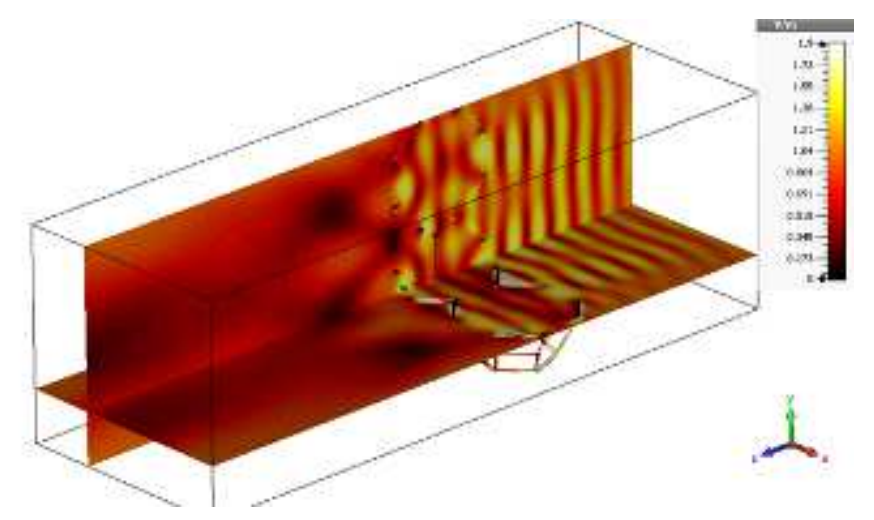

b)

Figure 6. Calculated amplitude of the electric field vector for carbon-based Kelvin cells having two strut thicknesses: (a) $d=0.5 \mathrm{~mm}$ and (b) $d=0.1 \mathrm{~mm}$. Numerical simulations were performed with a backbone dc conductivity of $20000 \mathrm{~S} \mathrm{~m}^{-1}$ and a strut length of $1 \mathrm{~mm}$.

Fig. 7 shows the dependence of the elements of the scattering matrix $S_{11}$ and $S_{12}$ calculated for cubic structures consisting of several layers of Gibson-Ashby cells in free space. From the analysis of the computer simulation, we can conclude that in the case of relatively low backbone electrical conductivity, the addition of more layers decreases the overall level of transmission, and the overall reflection is mainly determined by the reflection of the first carbon layer at the extreme surface of the structure. At the same time, for a structure based on a highly conductive skeleton, the addition of each new layer leads to the appearance of new resonances, and the electromagnetic response becomes more complex. The absorption level $A=1-\left(S_{11}\right)^{2}-\left(S_{21}\right)^{2}$ can reach up to $70 \%$ for a moderate electrical conductivity $\left(200 \mathrm{~S} \cdot \mathrm{m}^{-1}\right)$ near the resonance, and about $50 \%$ outside the resonance. Increasing the conductivity of the skeleton leads to almost 
perfect absorption at certain frequencies corresponding to cell and window sizes in the case of a single-layer structure.
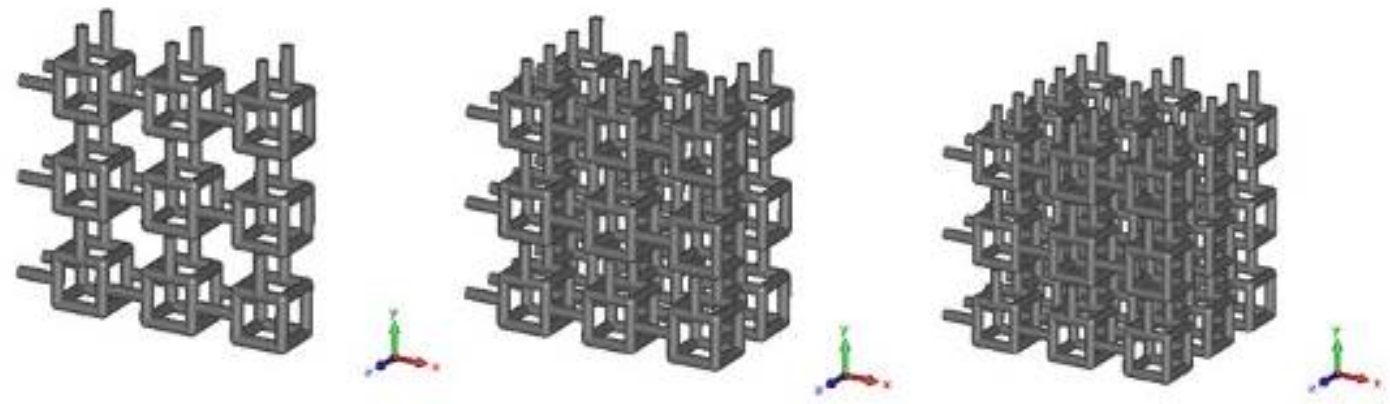

a)

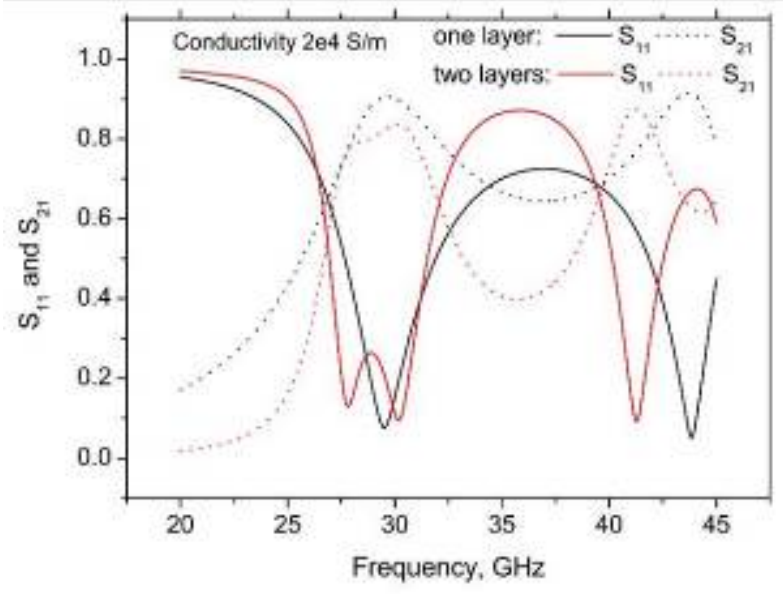

b)

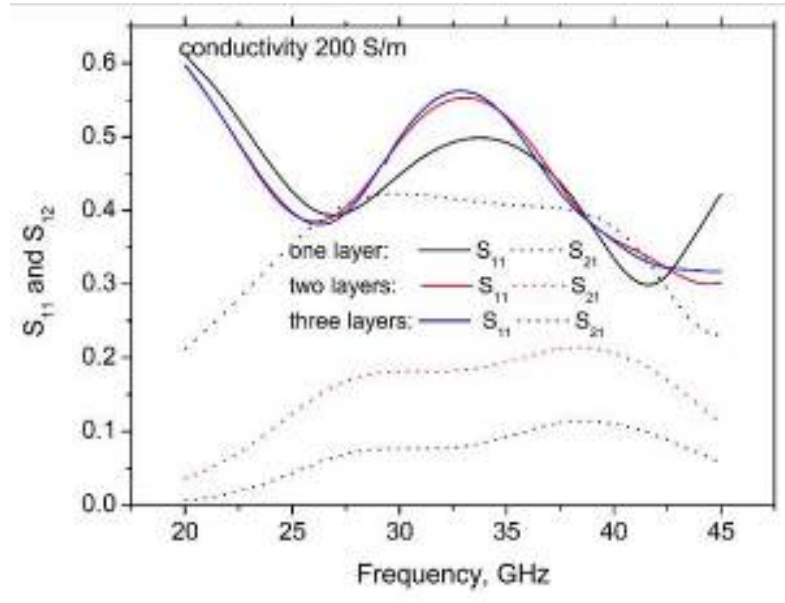

c)

Figure 7. Frequency dependence of $S_{11}$ and $S_{21}$ parameters for a 3D structure based on one, two, or three layers of Gibson-Ashby cells as pictured in (a), and for two different values of backbone dc conductivity: (b) $20000 \mathrm{~S} \cdot \mathrm{m}^{-1}$; and (c) $200 \mathrm{~S} \cdot \mathrm{m}^{-1}$. The following geometric parameters were used in the calculation: $l=3 \mathrm{~mm}$, and $d=0.83 \mathrm{~mm}$.

\subsection{Highly conductive skeleton: resonant properties and EM field concentration}

The electromagnetic response of 3D periodic structures based on Gibson-Ashby cells made of glassy carbon and measured in the microwave frequency range by the waveguide method is shown in Fig. 8. A good qualitative agreement between the experimental results and the results of computer simulation can be observed. The quantitative discrepancy is primarily due to the defects present in the real samples. As follows from the analysis of the field 
distribution, the increase in absorption near $35.6 \mathrm{GHz}$ is associated with interference at a given frequency.

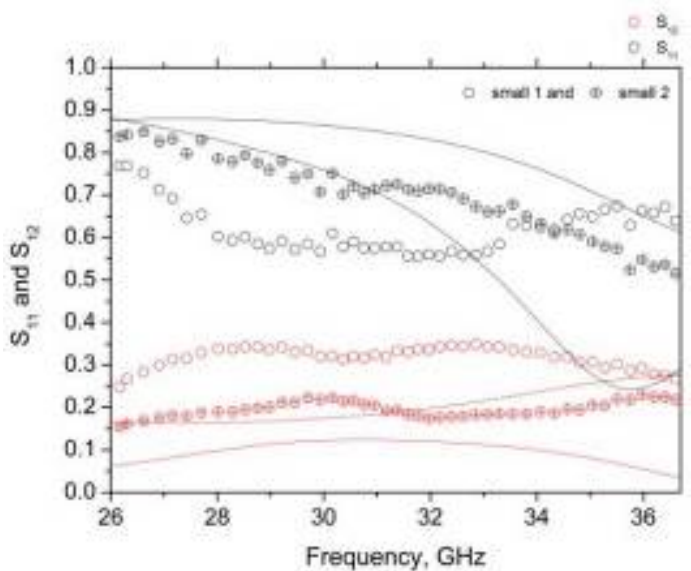

a)

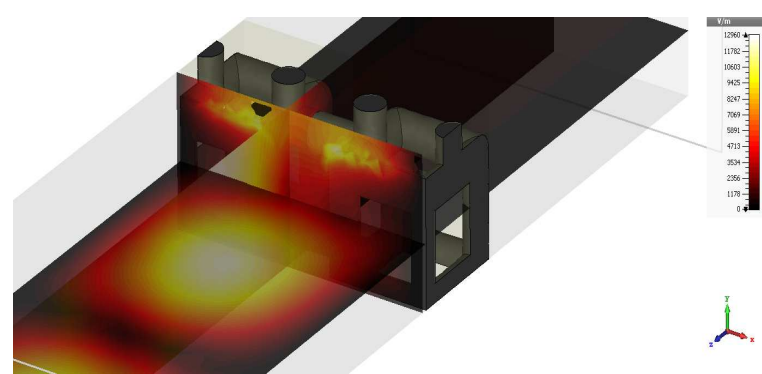

c)

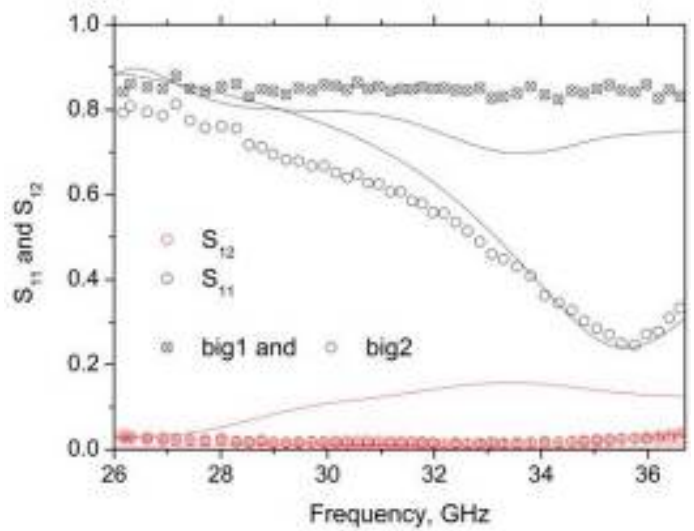

b)

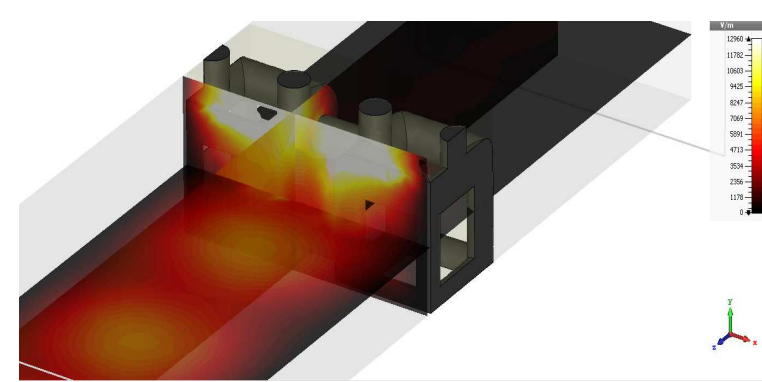

d)

Figure 8. Frequency dependence in the microwave range of the electromagnetic response of 3D structures based on: (a) one single layer; and (b) two layers of Gibson-Ashby cells made of glassy carbon. Samples 1 and 2 were cut along two perpendicular directions related to the symmetry axes. The points on the graph are experimental data, whereas the curves correspond to computer simulation results obtained with the following geometric parameters: $L=2.4 \mathrm{~mm}, a=$ $0.84 \mathrm{~mm}$, and dc skeleton conductivity of $1200 \mathrm{~S} \cdot \mathrm{m}^{-1}$. (c) and (d) Distribution of the electromagnetic field in the waveguide, calculated for the frequency of $26 \mathrm{GHz}$ and $35.6 \mathrm{GHz}$ and corresponding to cases (a) and (b), respectively. 


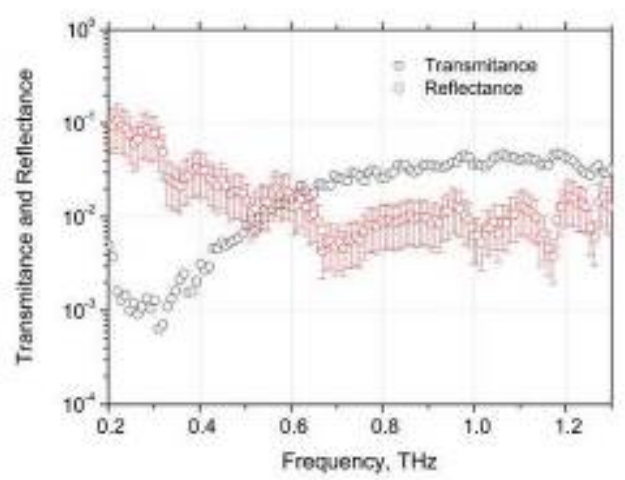

a)

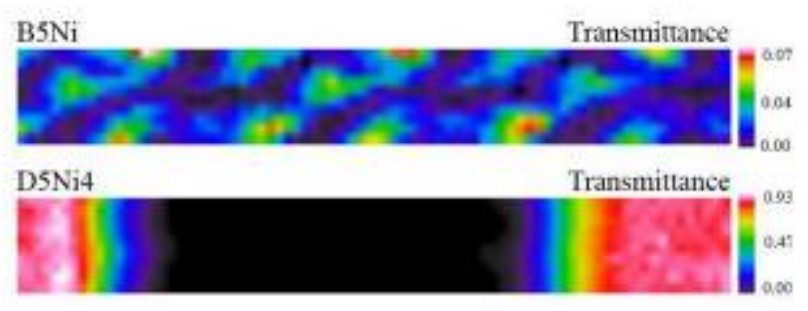

c)

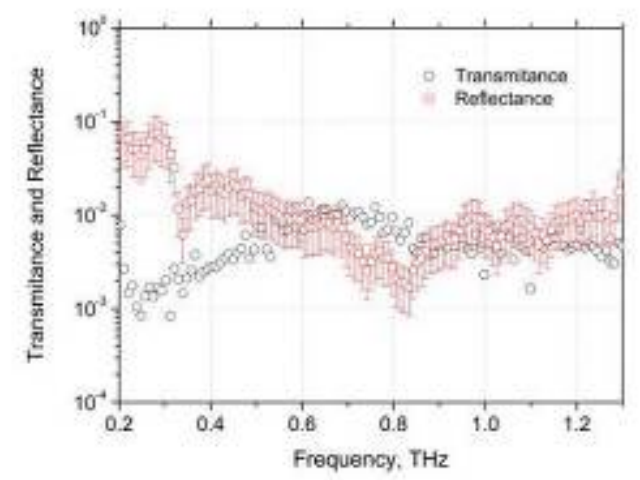

b)

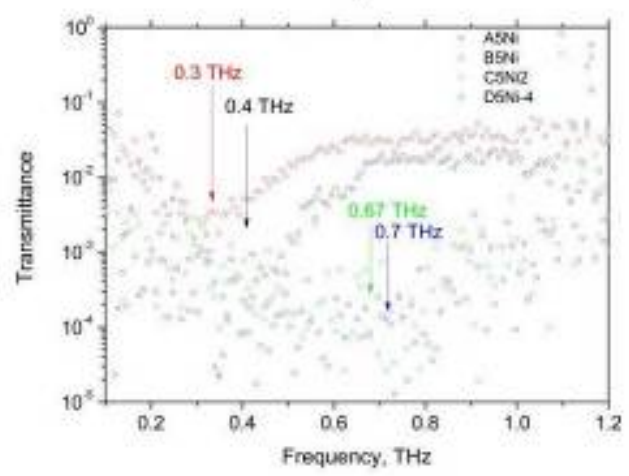

d)

Figure 9. Frequency dependence of transmission $(T)$ and reflection $(R)$ coefficients, measured for two positions of the $\mathrm{THz}$ beam on the sample of 3D glassy carbon structure having the largest Kelvin cell size (B5Ni): (a) position \#1 and (b) position \#2 corresponding to the $\mathrm{THz}$ spot in the centre of the cell and at the edge, respectively. (c) $2 \mathrm{D}$ scan at $0.8 \mathrm{THz}$ along the surface of the (left) B5Ni sample and the sample having the smallest cell size, D5Ni4 (right). (d) Frequency dependence of the transmission coefficient of samples having different Kelvin cell sizes.

Fig. 9a and $9 \mathrm{~b}$ show how the reflection and transmission coefficients measured in the $\mathrm{THz}$ frequency range change as a function of frequency for the 3D cubic structures whose geometric characteristics have been reported in Table 1. In spite of high scattering of raw reflection data (Fig.9a,b), the contribution of reflectance of carbon meshes to the overall electromagnetic response of glassy carbon Kelvin cells made mesh is negligible, close to 0. Together with close to 0 transition we approach to the perfect absorption of electromagnetic radiation in $\mathrm{THz}$ range by highly conductive carbon architectures. 
Since the characteristic $\mathrm{THz}$ beam size is $4 \mathrm{~mm}$, i.e., comparable to the dimensions of a unit cell in the case of $\mathrm{B} 5 \mathrm{Ni}$ sample, then the electromagnetic response of the latter depends on the position of the $\mathrm{THz}$ beam on the sample. There is a certain cut-off frequency, around 0.4 and 0.3 $\mathrm{THz}$ well pronounced for samples of A- and B-series, respectively, above which the transmission coefficient increases (see Fig. 9d). By decreasing the cell size, this frequency shifts towards higher values, as seen in Fig. 9d. For C- and D-series the estimated values of cut-off frequency are around 0.67 and $0.70 \mathrm{THz}$ based on their scale factor compared to the A- and B-series samples. 2D scans measured at $0.8 \mathrm{THz}$, shown in Fig. 9c, support the idea of a possible periodical concentration of electromagnetic field by using lossy photonic crystals based on a highly conductive backbone.

The use of time-resolved spectroscopy techniques allows estimating the delay of the reflected signal. Fig. 10 thus shows the time dependence of the reflected THz pulse in the case of an ideal reflector (a golden mirror) and for a structure based on glassy carbon Kelvin cells having different sizes. One can see that there is no time delay for the D5Ni4 sample, which may be an indication that the main contribution to the reflection comes from the extreme surface (Fig. 10b). At the same time, for a series of larger B5Ni samples, the $\mathrm{THz}$ delay of the reflection pulse is about 17 ps (Fig. 10a), which indicates that multiple reflection from the internal structure of the sample also contributes to the overall reflection.

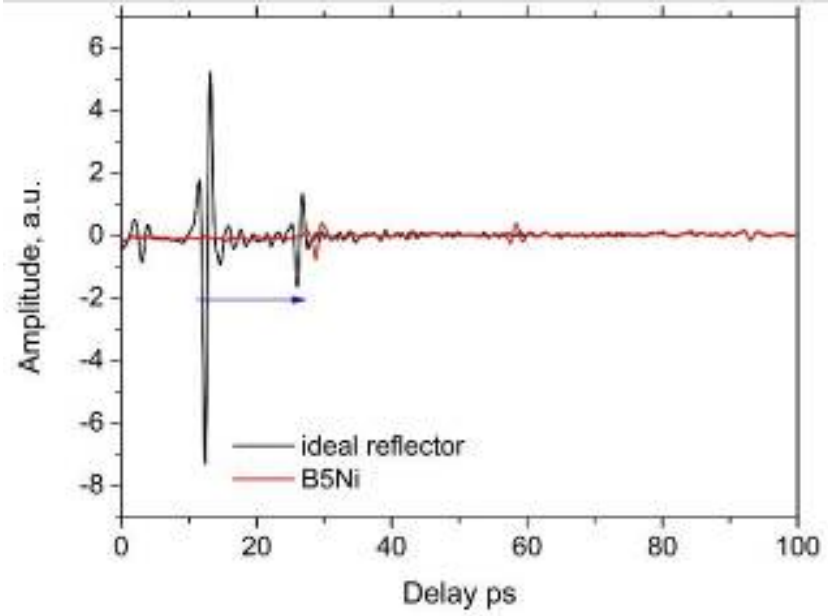

a)

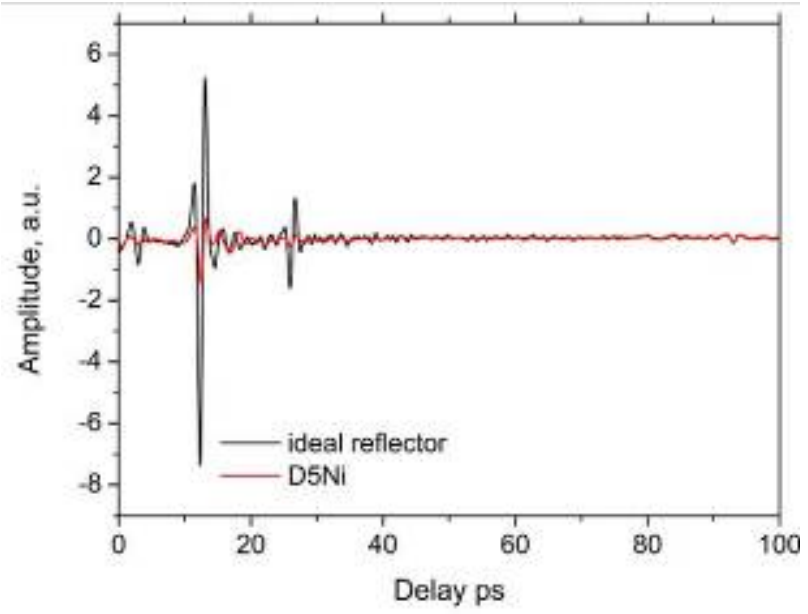

b) 
Figure 10. Impulse of the reflected $\mathrm{THz}$ signal for the case of an ideal reflector (black line) and for 3D glassy carbon periodic structures based on: (a) large (B5Ni); and (b) small (D5Ni4) Kelvin cells (the arrow indicates the time delay).

\section{Conclusion}

The comparative studies of the EM response of 3D periodic structures made of carbon-based backbone of either high or low-to-medium electrical conductivity have brought several important application conclusions. First, the most unexpected finding is that a highly conductive backbone is not necessary for getting high and almost perfect absorption. Intermediate or even poor conductivity, e.g. $10-30 \mathrm{~S} \cdot \mathrm{m}^{-1}$ for the geometry and lattice parameters considered in the present work is indeed enough to reach more that $85 \%$ of absorption in a wide frequency range. This finding also suggests that, based on a backbone of given conductivity, one might always find, through a preliminary modelling step, the most relevant lattice parameters allowing a 3D-printed cellular structure to present either high broadband or perfect resonant absorption. This is a very significant result considering that the conductivity of the material (either in the case of approach

(i) in Fig. 1a, glassy carbon, or in the case of approach (ii) in Fig. 1b, composite filament, is the most difficult to tune).

Another good point is that substantial changes in the backbone conductivity (e.g. in the case of the presently examined cubic geometry, from 5 to $40 \mathrm{~S} \cdot \mathrm{m}^{-1}$ ) don't produce significant changes in the EM response. This is an important result when it comes to obtaining a robust technology transfer from lab to mass production not requiring time-consuming optimization steps, since it allows a not so strict control of the filament conductivity without lowering the EM performances of the final 3D-printed products. However, the low-to-medium conductivity of the 3D mesh does not allow finely tuning the EM response of the structure by impregnation with a dielectric medium. 
Another way of adjusting the EM properties of 3D-printed structure would thus be to use the FDM technique with composite filaments. Glassy carbon-based monoliths are indeed rigid and cannot be reversibly deformed, whereas the cells and windows sizes responsible for the resonance position would be modulated in composite-based photonic crystals made of flexible and stretchable matrix, with which tuneability would be obtained through mechanical deformation.

In conclusion, high although not perfect broadband absorption is possible for 3D-printed cellular structures whose backbone is not highly conductive, i.e., in the range $1-30 \mathrm{~S} \cdot \mathrm{m}^{-1}$, whereas resonant perfect tuneable absorption can be achieved by $3 \mathrm{D}$ meshes made of highly conductive glassy carbon. Periodic concentration of EM waves is possible with highly conductive 3D architectures, the resonant frequency being determined by cell and windows sizes. Using one or the other approach for getting pre-defined required EM performances depends on the intended application.

\section{Acknowledgements}

This research was sponsored by the NATO Science for Peace and Security Programme [grant G5697 CERTAIN “Globular carbon-based structures and metamaterials for enhanced electromagnetic protection"], and by Horizon 2020 EC [grant H2020 RISE 734164 Graphene 3D]. PK is thankful to Academy of Finland Flagship Programme [grant 320166 PREIN "Photonics Research and Innovation"], and Horizon 2020 EC [grant H2020 IF TURANDOT 836816]. We are thankful to Prof. Donald M. Migliore (University of Cassino and Southern Lazio) for supporting with CST Studio calculation, and to Dr. Jan Macutkevic (Vilnius University) for valuable discussions. DB is thankful for support by Tomsk State University Competitiveness Improvement Program. AC and VF gratefully acknowledge the financial support of FEDER [TALiSMAN project (2019-000214)]. 


\section{References}

1. F. Qin and C. Brosseau, A review and analysis of microwave absorption in polymer composites filled with carbonaceous particles, Journal of Applied Physics, 111, (2012) 061301, https://doi.org/10.1063/1.3688435

2. K.N. Rozanov, Ultimate thickness to bandwidth ratio of radar absorbers, IEEE Transactions on Antennas and Propagation, 48(8) (2000) 1230 - 1234, 10.1109/8.884491

3. Z. Fang, C. Li, J. Sun, H. Zhang, J. Zhang, The electromagnetic characteristics of carbon foams, Carbon, 45(15) (2007) 2873-9, 10.1016/j.carbon.2007.10.013

4. Z. Chen, C. Xu, C. Ma, W. Ren, H-M. Cheng, Lightweight and Flexible Graphene Foam Composites for High-Performance Electromagnetic Interference Shielding, Adv. Mater., 25(9) (2013) 1296-300, 10.1002/adma.201204196

5. M. Letellier, J. Macutkevic, P. Kuzhir, J. Banys, V. Fierro, A. Celzard, Electromagnetic properties of model vitreous carbon foams, Carbon, 122 (2017) 217-227, $\underline{10.1016 / \text { j.carbon.2017.06.080 }}$

6. K. Batrakov, P. Kuzhir, S. Maksimenko, A. Paddubskaya, S. Voronovich, Ph. Lambin, T. Kaplas, Yu. Svirko, Flexible transparent graphene/polymer multilayers for efficient electromagnetic field absorption, Scientific Reports, 4 (2014) 7191, doi:10.1038/srep07191

7. Y. Ra'di, C. R. Simovski, and S. A. Tretyakov, Thin Perfect Absorbers for Electromagnetic Waves: Theory, Design, and Realizations, Phys. Rev. Applied, 3 (2015) 037001, https://doi.org/10.1103/PhysRevApplied.3.037001

8. S. Bauer, S. Bauer-Gogonea, B. Ploss, The physics of pyroelectric infrared devices, Appl. Phys. B54 (1992) 544-551, https://doi.org/10.1007/BF00325524 
9. A. Paddubskaya, M. Demidenko, K. Batrakov, G. Valušis, T. Kaplas, Yu. Svirko, P. Kuzhir, Tunable Perfect THz Absorber Based on a Stretchable Ultrathin Carbon-Polymer Bilayer, Materials, 12 (2019) 143, doi:10.3390/ma12010143

10. S. Y. Lin, J. G. Fleming, D. L. Hetherington, B. K. Smith, R. Biswas, K. M. Ho, M. M. Sigalas, W. Zubrzycki, S. R. Kurtz, J. Bur, A three-dimensional photonic crystal operating at infrared wavelengths, Nature, 394 (1998) 251-253, https://doi.org/10.1038/28343

11. E. Cubukcu, K. Aydin, E. Ozbay, S. Foteinopoulou, C.M. Soukoulis, Electromagnetic waves: Negative refraction by photonic crystals, Nature, 423 (2003) 604-5, $\underline{10.1038 / 423604 b}$

12. N. N. Lepeshkin, A. Schweinsberg, G. Piredda, R.S. Bennink, R. W. Boyd, Enhanced Nonlinear Optical Response of One-Dimensional Metal-Dielectric Photonic Crystals, Phys. Rev. Lett. 93 (2004) 123902, https://doi.org/10.1103/PhysRevLett.93.123902

13. K. Busch, S. Lölkes, R. B. Wehrspohn, H. Föll, Photonic Crystals: Advances in Design, Fabrication, and Characterization, ISBN: 978-3-527-60717-4, Wiley, 2006

14. K. Yoshino, Y. Shimoda, Y. Kawagishi, K. Nakayama, M. Ozaki, Temperature tuning of the stop band in transmission spectra of liquid-crystal infiltrated synthetic opal as tunable photonic crystal, Appl. Phys. Lett. 75 (1999) 932-4, https://doi.org/10.1063/1.124558

15. A. Paddubskaya, N. Valynets, P. Kuzhir, K. Batrakov, S. Maksimenko, R.Kotsilkova, H. Velichkova, I. Petrova, I. Biró, K. Kertész, G. I. Márk, Z. E. Horváth, L. P. Biró, Electromagnetic and Thermal properties of 3D Printed Multilayered Nano-carbon / Poly(lactic) Acid Structures, Journal of Applied Physics, 119, (2016) 135102, doi: $\underline{10.1063 / 1.4945576}$

16. J. Ren, J. Yuan Yin, 3D-Printed Low-Cost Dielectric-Resonator-Based Ultra-Broadband Microwave Absorber Using Carbon-Loaded Acrylonitrile Butadiene Styrene Polymer, Materials (Basel), 11(7) (2018) E1249, doi: 10.3390/ma11071249 
17. A. Szczurek; A. Ortona; L. Ferrari; E. Rezaei; G. Medjahdi; V. Fierro; D. Bychanok; P. Kuzhir; A. Celzard, Carbon periodic cellular architectures, Carbon, 88 (2015) 70-85, doi:10.1016/j.carbon.2015.02.069

18. X. Wei, D. Li, W. Jiang, Zh. Gu, X. Wang, Z. Zhang, Zh. Sun, 3D Printable Graphene Composite, Scientific Reports, 5 (2015) 11181, doi: 10.1038/srep11181

19. D. Bychanok, P. Angelova, A. Paddubskaya, D. Meisak, L. Shashkova, M. Demidenko, A. Plyushch, E. Ivanov, R. Krastev, R. Kotsilkova, F. Y. Ogrin, P. Kuzhir, Terahertz absorption peak in graphene nanoplatelets/polylactic acid composites: effects of fine dispersion, agglomeration and percolation, J. Phys. D: Appl. Phys., 51 (2018) 145307 https://doi.org/10.1088/1361-6463/aab1a5

20. A. E. Vandenbosch, A. Vasylchenko, A Practical Guide to 3D Electromagnetic Software Tools, Published: April 4th 2011, DOI: 10.5772/14756

21. S. Duan, K. Yang, Z. Wang, M. Chen, L. Zhang, H. Zhang, Ch. Li, Fabrication of Highly Stretchable Conductors Based on 3D Printed Porous Poly(dimethylsiloxane) and Conductive Carbon Nanotubes/Graphene Network, ACS Appl. Mater. Interfaces, 8 (3) (2016) 2187-2192, https://doi.org/10.1021/acsami.5b10791 